\title{
Copper-64-labeled antibody fragments for immuno- PET/radioimmunotherapy with low renal radioactivity levels and amplified tumor-kidney ratios
}

Hiroyuki Suzuki, Shota Kise, Yuta Kaizuka, Reo Watanabe, Tsubasa Sugawa, Takako Furukawa, Hirofumi Fujii, Tomoya Uehara*

Hiroyuki Suzuki; Laboratory of Molecular Imaging and Radiotherapy, Graduate School of Pharmaceutical Sciences, Chiba University, 1-8-1 Inohana, Chuo-ku, Chiba, Chiba, 260-8675 Japan

Shota Kise; Laboratory of Molecular Imaging and Radiotherapy, Graduate School of Pharmaceutical Sciences, Chiba University, 1-8-1 Inohana, Chuo-ku, Chiba, Chiba, 260-8675 Japan

Yuta Kaizuka; Laboratory of Molecular Imaging and Radiotherapy, Graduate School of Pharmaceutical Sciences, Chiba University, 1-8-1 Inohana, Chuo-ku, Chiba, Chiba, 260-8675 Japan

Reo Watanabe; Laboratory of Molecular Imaging and Radiotherapy, Graduate School of Pharmaceutical Sciences, Chiba University, 1-8-1 Inohana, Chuo-ku, Chiba, Chiba, 260-8675 Japan

Tsubasa Sugawa; Laboratory of Molecular Imaging and Radiotherapy, Graduate School of Pharmaceutical Sciences, Chiba University, 1-8-1 Inohana, Chuo-ku, Chiba, Chiba, 260-8675 Japan

Takako Frukawa; Nagoya University Graduate School of Medicine, 1-1-20 Daiko-Minami, Higashi-ku, Nagoya, Aichi, 4618673, Japan

Hirofumi Fujii; Division of Functional Imaging, Exploratory Oncology Research and Clinical Trial Center, National Cancer Center, 6-5-1 Kashiwanoha, Kashiwa, Chiba, 277-8577, Japan

Tomoya Uehara; Laboratory of Molecular Imaging and Radiotherapy, Graduate School of Pharmaceutical Sciences, Chiba University, 1-8-1 Inohana, Chuo-ku, Chiba, Chiba, 260-8675 Japan

\section{List of Supplementary Materials}

HPLC method

Table S1. Biodistribution of radioactivity in normal mice after injection of $\left[{ }^{64} \mathrm{Cu}\right] \mathrm{Cu}-\mathrm{NOTA}-\mathrm{MVK}-\mathrm{Fab},\left[{ }^{64} \mathrm{Cu}\right] \mathrm{Cu}-\mathrm{NOTA}-\mathrm{Fab}$ and $\left[{ }^{67} \mathrm{Ga}\right] \mathrm{Ga}-\mathrm{NOTA}-\mathrm{MVK}-\mathrm{Fab}$.

Table S2. Biodistribution of radioactivity in nude mice bearing MC-38 cells after $3 \mathrm{~h}$ injection of $\left[{ }^{64} \mathrm{Cu}\right] \mathrm{Cu}-\mathrm{NOTA}-\mathrm{MVK}-\mathrm{Fab}$ and $\left[{ }^{64} \mathrm{Cu}\right] \mathrm{Cu}-\mathrm{NOTA}-\mathrm{Fab}$.

Figure S1 SE-HPLC profiles of $\left[{ }^{64} \mathrm{Cu}\right] \mathrm{Cu}-\mathrm{NOTA}-\mathrm{MVK}-\mathrm{Fab},\left[{ }^{64} \mathrm{Cu}\right]-\mathrm{NOTA}-\mathrm{Fab}$ and $\left[{ }^{67} \mathrm{Ga}\right] \mathrm{Ga}-\mathrm{NOTA}-\mathrm{MVK}-\mathrm{Fab}$. 


\section{HPLC methods}

General. Analytical reversed phase HPLC (RP-HPLC) was performed with a Cosmosil 5C18-AR-300 column (4.6 mm $\times 150 \mathrm{~mm}$, Nacalai Tesque Inc., Kyoto, Japan) at a flow rate of $1 \mathrm{~mL} / \mathrm{min}$ using a gradient mobile phase starting from $95 \%$ A $(0.1 \%$ aqueous trifluoroacetic acid (TFA)) and 5\% B (acetonitrile with 0.1\% TFA) to 60\% A and 40\% B in 30 min. The eluent was monitored with an online UV/Visible single beam detector at $254 \mathrm{~nm}$ (L-7405, Hitachi Co. Ltd., Tokyo, Japan) coupled to a NaI(Tl) radioactivity detector (Gabi Star, Raytest, Strubenhardt, Germany). TLC analyses were performed with RP18 plates (Silica gel 60 RP-18 F254S, Merck Ltd., Tokyo) developed with $0.1 \mathrm{M}$ ammonium acetate/methanol (1/1). Radioactivity was measured using a $\gamma$-miniGITA TLC Scanner (Raytest) and an automated gamma well counter. 
Table S1. Biodistribution of radioactivity in normal mice after injection of $\left[{ }^{64} \mathrm{Cu}\right] \mathrm{Cu}-\mathrm{NOTA}-\mathrm{MVK}-\mathrm{Fab},\left[{ }^{64} \mathrm{Cu}\right] \mathrm{Cu}-\mathrm{NOTA}-$ Fab and $\left[{ }^{67} \mathrm{Ga}\right] \mathrm{Ga}-N O T A-M V K-F a b$.

\begin{tabular}{|c|c|c|c|c|c|c|c|c|c|c|c|c|}
\hline \multirow[b]{4}{*}{ Blood } & \multicolumn{12}{|c|}{ Time after injection } \\
\hline & \multicolumn{3}{|c|}{$10 \mathrm{~min}$} & \multicolumn{3}{|c|}{$1 \mathrm{~h}$} & \multicolumn{3}{|c|}{$3 \mathrm{~h}$} & \multicolumn{3}{|c|}{$6 \mathrm{~h}$} \\
\hline & \multicolumn{12}{|c|}{$\left[{ }^{64} \mathrm{Cu}\right] \mathrm{Cu}-\mathrm{NOTA}-\mathrm{MVK}-\mathrm{Fab}$} \\
\hline & 42.0 & \pm & 1.65 & 25.7 & \pm & 2.54 & 17.7 & \pm & 7.02 & $8.28^{\mathrm{a}}$ & \pm & 0.88 \\
\hline Liver & 8.34 & \pm & 0.82 & 6.79 & \pm & 0.36 & $5.37^{\mathrm{b}}$ & \pm & 0.39 & $4.85^{\mathrm{b}}$ & \pm & 1.12 \\
\hline Kidney & 21.9 & \pm & 1.05 & $22.3^{\mathrm{a}}$ & \pm & 1.79 & $15.7^{\mathrm{a}}$ & \pm & 2.12 & $11.0^{\mathrm{a}}$ & \pm & 0.71 \\
\hline Spleen & 8.81 & \pm & 1.05 & 11.3 & \pm & 1.42 & $11.6^{\mathrm{b}}$ & \pm & 1.23 & 12.2 & \pm & 4.46 \\
\hline Stomach* & 0.37 & \pm & 0.07 & 0.50 & \pm & 0.03 & $1.01^{\mathrm{b}}$ & \pm & 0.15 & 0.88 & \pm & 0.24 \\
\hline Intestine* & 2.08 & \pm & 0.37 & 4.61 & \pm & 0.44 & 7.98 & \pm & 0.99 & 15.3 & \pm & 6.93 \\
\hline Urine* & & & & & & & & & & 29.0 & \pm & 1.96 \\
\hline \multirow[t]{2}{*}{ Feces* } & & & & & & & & & & 0.60 & \pm & 0.13 \\
\hline & \multicolumn{12}{|c|}{$\left[{ }^{64} \mathrm{Cu}\right] \mathrm{Cu}-\mathrm{NOTA}-\mathrm{Fab}$} \\
\hline Blood & 40.2 & \pm & 5.65 & 22.1 & \pm & 2.28 & 12.3 & \pm & 0.59 & 6.70 & \pm & 0.88 \\
\hline Liver & 8.05 & \pm & 0.95 & 5.81 & \pm & 1.03 & 5.44 & \pm & 0.52 & 5.23 & \pm & 1.59 \\
\hline Kidney & 26.5 & \pm & 3.24 & 57.0 & \pm & 12.71 & 70.15 & \pm & 5.00 & 63.3 & \pm & 19.9 \\
\hline Spleen & 11.7 & \pm & 1.07 & 11.2 & \pm & 2.25 & 10.8 & \pm & 1.98 & 15.0 & \pm & 8.72 \\
\hline Stomach* & 0.38 & \pm & 0.21 & 0.50 & \pm & 0.17 & 0.60 & \pm & 0.09 & 0.54 & \pm & 0.09 \\
\hline Intestine* & 1.78 & \pm & 0.19 & 3.35 & \pm & 0.28 & 4.01 & \pm & 0.32 & 4.29 & \pm & 0.23 \\
\hline Urine* & & & & & & & & & & 15.3 & \pm & 2.57 \\
\hline \multirow[t]{2}{*}{ Feces* } & & & & & & & & & & 0.11 & \pm & 0.04 \\
\hline & \multicolumn{12}{|c|}{$\left[{ }^{67} \mathrm{Ga}\right] \mathrm{Ga}-\mathrm{NOTA}-\mathrm{MVK}-\mathrm{Fab}$} \\
\hline Blood & 44.9 & \pm & 1.34 & 24.7 & \pm & 2.58 & 15.6 & \pm & 2.05 & 9.22 & \pm & 1.25 \\
\hline Liver & 10.4 & \pm & 1.40 & 7.64 & \pm & 0.91 & 8.06 & \pm & 1.62 & 6.65 & \pm & 0.92 \\
\hline Kidney & 23.0 & \pm & 2.77 & 18.4 & \pm & 1.73 & 13.8 & \pm & 1.63 & 10.6 & \pm & 0.97 \\
\hline Spleen & 10.5 & \pm & 0.70 & 8.65 & \pm & 1.67 & 8.14 & \pm & 1.17 & 8.19 & \pm & 1.36 \\
\hline Stomach* & 0.31 & \pm & 0.04 & 0.58 & \pm & 0.16 & 0.67 & \pm & 0.22 & 0.41 & \pm & 0.08 \\
\hline Intestine* & 2.43 & \pm & 0.43 & 4.13 & \pm & 0.64 & 7.11 & \pm & 0.65 & 11.9 & \pm & 0.28 \\
\hline Urine* & & & & & & & & & & 25.3 & \pm & 3.98 \\
\hline Feces* & & & & & & & & & & 1.97 & \pm & 1.82 \\
\hline
\end{tabular}

*Expressed as \%ID

Significance determined by one-way analysis of variance followed by Tukey's multiple-comparison test.

${ }^{a} P<0.05$ compared to $\left[{ }^{64} \mathrm{Cu}\right] \mathrm{Cu}-\mathrm{NOTA}-\mathrm{Fab}$

${ }^{b} P<0.05$ compared to $\left[{ }^{67} \mathrm{Ga}\right] \mathrm{Ga}-\mathrm{NOTA}-\mathrm{MVK}-\mathrm{Fab}$ 
Table S2. Biodistribution of radioactivity in nude mice bearing MC-38 cells after 3 h injection of [ $\left.{ }^{64} \mathrm{Cu}\right] \mathrm{Cu}-\mathrm{NOTA}-\mathrm{MVK}-\mathrm{Fab}$ and $\left[{ }^{64} \mathrm{Cu}\right] \mathrm{Cu}-\mathrm{NOTA}-\mathrm{Fab}$.

\begin{tabular}{|c|c|c|c|c|c|c|}
\hline \multicolumn{2}{|c|}{ Tissue radioactivity is expressed as \%ID/g [for each group, $\mathrm{n}=5$; results are reported as mean \pm SD] } \\
\hline & \multicolumn{2}{|c|}{$\left[{ }^{64} \mathrm{Cu}\right] \mathrm{Cu}-\mathrm{NOTA}-\mathrm{MVK}-\mathrm{Fab}$} & \multicolumn{3}{|c|}{$\left[{ }^{64} \mathrm{Cu}\right] \mathrm{Cu}-\mathrm{NOTA}-\mathrm{Fab}$} \\
\hline Blood & 14.0 & \pm & 0.93 & 5.61 & \pm & 0.56 \\
\hline Liver & 5.28 & \pm & 0.44 & 71.0 & \pm & 5.17 \\
\hline Kidney & $17.8^{\mathrm{a}}$ & \pm & 2.03 & 9.06 & \pm & 0.74 \\
\hline Spleen & 9.54 & \pm & 1.16 & 2.12 & \pm & 0.75 \\
\hline Muscle & 2.49 & \pm & 1.10 & 0.56 & \pm & 0.11 \\
\hline Stomach* & 0.82 & \pm & 0.23 & 3.46 & \pm & 0.45 \\
\hline Intestine* & $7.68^{\mathrm{a}}$ & \pm & 0.72 & 5.89 & \pm & 1.44 \\
\hline Tumor & 5.51 & \pm & 0.78 & 0.54 & \pm & 0.12 \\
\hline Tumor/Blood & 0.39 & \pm & 0.05 & 3.07 & \pm & 1.25 \\
\hline Tumor/Muscle & 3.08 & \pm & 2.61 & & \\
\hline
\end{tabular}

*Expressed as \%ID

Significance determined by one-way analysis of variance followed by Tukey’s multiple-comparison test.

${ }^{a} P<0.05$ compared to $\left[{ }^{64} \mathrm{Cu}\right] \mathrm{Cu}-\mathrm{NOTA}-\mathrm{Fab}$ 


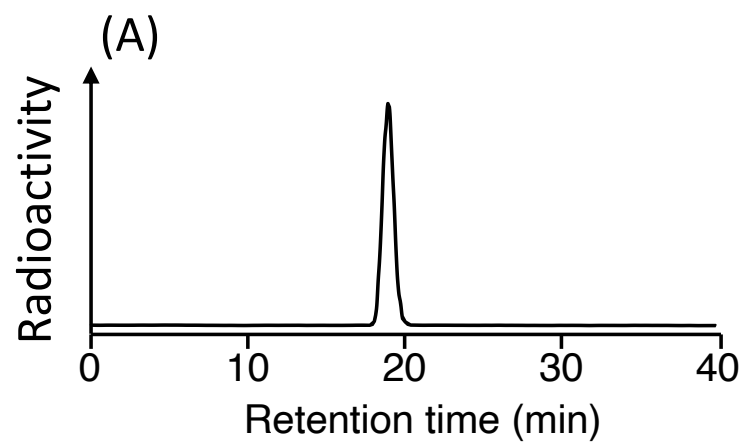

(B)

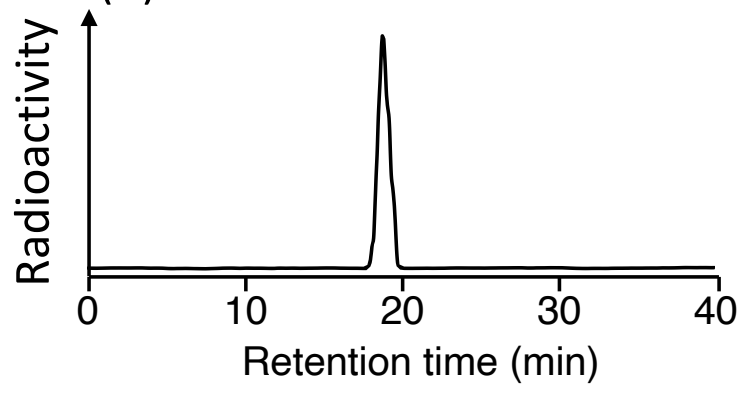

Figure S1. SE-HPLC profiles of $\left[{ }^{64} \mathrm{Cu}\right] \mathrm{Cu}-\mathrm{NOTA}-\mathrm{MVK}-\mathrm{Fab}(\mathrm{A})$ and $\left[{ }^{64} \mathrm{Cu}\right]-\mathrm{NOTA}-\mathrm{Fab}(\mathrm{B})$. 